\title{
ESTUDIO TAXONOMICO DE CUATRO ESPECIES DE ENCINO (QUERCUS) DESCRITAS POR WARBURG
}

\author{
Ma. de Lourdes Aguilar Enriquez \\ Y \\ Silvia Romero Rangel \\ Herbario de la Escuela Nacional de Estudios Profesionales, Iztacala \\ Universidad Nacional Autónoma de México \\ Apartado Postal 314 \\ 54090 Tlalnepantla, Edo. de México, México
}

\section{RESUMEN}

Se determinaron como sinónimos de Quercus hintonii Warb. a Q. apiophylla Warb., Q. ochroestes Warb. y $Q$. sagata Warb., con base en el análisis de las descripciones originales, fototipos y ejemplares existentes en los herbarios. Además se realizaron observaciones de las poblaciones y colectas de ejemplares en las localidades tipo.

\section{ABSTRACT}

Q. apiophylla Warb., Q. ochroestes Warb. and Q. sagata Warb. were determined as synonyms of $Q$. hintonii Warb. on the basis of analysis of original descriptions, phototypes, and specimens in the herbaria; furthermore, observations of populations and collections of specimens were done in the type localities.

\section{INTRODUCCION}

Durante el desarrollo del proyecto "Encinos del Estado de México" se tuvo dificultad en la determinación de algunos ejemplares de este género colectados en el suroeste de la entidad, principalmente en los municipios de Tejupilco y Temascaltepec. Después de consultar la bibliografía existente (Martínez, 1954) observamos que los especímenes colectados tienen afinidad con Quercus hintonii, Q. sagata, Q. ochroestes y $Q$. apiophylla. Estas especies resultan difíciles de distinguir entre sí, lo que nos llevó a la necesidad de esclarecer su situación taxonómica y nomenclatural.

Warburg (1939) describió ocho especies de encinos con base en material colectado por G. B. Hinton de 1931 a 1939 en diferentes localidades del Estado de México y depositado en el Herbario de Kew, Inglaterra, a mencionar: Quercus paradifolia, Q. platyphylla, Q. atriglans, Q. dolichopus, Q. hintonii, $Q$. apiophylla, $Q$. ochroestes y $Q$. sagata. Las primeras cuatro han sido consideradas por Muller y McVaugh (1972) como sinónimos de los siguientes taxa: 
1. Quercus elliptica Née, An. Ci. Nat. 3: 278. 1801. Q. paradifolia Warb., Kew Bull. 1939: 92. 1939.

2. Quercus magnoliifolia Née, An. Ci. Nat. 3: 268. 1801. Q. platyphylla Warb., Kew Bull. 1939: 85. 1939.

3. Quercus obtusata Humb. \& Bonpl., PI. Aequinoct 2: 76.1809. Q. atriglans Warb., Kew Bull. 1939: 88. 1939.

4. Quercus peduncularis Née, An. Ci. Nat. 3: 270.1801. Q. dolichopus Warb., Kew Bull. 1939: 87. 1939.

Las cuatro especies restantes son el objeto de este estudio.

\section{METODOLOGIA}

Se consultaron los herbarios de la Escuela Nacional de Ciencias Biológicas (ENCB), del Instituto de Biología (MEXU), de la Comisión Exploradora del Estado de México (CODAGEM), del Herbario Nacional Forestal (INIF) y de la Escuela Nacional de Estudios Profesionales Iztacala (IZTA). Se obtuvieron del Herbario Kew en Inglaterra las descripciones originales y fotografías de los tipos, estas últimas se encuentran depositadas en el Herbario Nacional Forestal (INIF).

Se visitaron los sitios referidos por Warburg (1939) para observar las poblaciones de encinos y colectar material, mismo que también se obtuvo de otras localidades cercanas en los municipios de Tejupilco, Amatepec, San Simón de Guerrero, Sultepec y Tlatlaya. Estos ejemplares se encuentran depositados en los herbarios de la Escuela Nacional de Estudios Profesionales Iztacala (IZTA), de la Escuela Nacional de Ciencias Biológicas (ENCB) y del Instituto de Biología (MEXU).

\section{RESULTADOS Y DISCUSION}

La consulta de los herbarios reveló que dos de las especies en estudio, Quercus apiophylla y $Q$. ochroestes, no estaban representadas en los mismos. También se encontró que unos ejemplares de $Q$. hintonii con hojas de borde entero estaban determinados como Q. dysophylla y otros, con hojas de borde dentado, lo estaban como Q. crassifolia. Estas tres especies se distinguen por los caracteres señalados en el Cuadro 1.

La comparación de las descripciones originales muestra que no existen diferencias claras entre las cuatro especies (Cuadro 2). Además, el análisis de los fototipos indica semejanza entre las especies estudiadas en cuanto a las características de hoja, fruto y lo que se puede percibir del aspecto general de la pubescencia.

Cabe observar que el espécimen correspondiente al tipo de $Q$. ochroestes Warb. se había determinado primeramente como Q. candicans Née, quizás porque el tomento de sus ramas jóvenes es parecido al de esta especie. Ejemplares con el mismo aspecto que el tipo fueron colectados por S. Romero, L. Aguilar y C. Rojas bajo los números 3213 , 3218 (IZTA).

Una situación similar se presentó en el tipo de $Q$. sagata Warb., pues en un principio se había identificado como $Q$. fournieri Trel.; actualmente $Q$. fournieri se considera sinónimo de $Q$. dysophylla. 
Cuadro 1. Caracteres distintivos entre $Q$. hintonii, $Q$. crassifolia y $Q$. dysophylla.

\begin{tabular}{|c|c|c|c|}
\hline & Q. hintonii & Q. crassifolia & Q. dysophylla \\
\hline Forma de la hoja & $\begin{array}{l}\text { ovada, lanceolada } \\
\text { o elíptica }\end{array}$ & obovada & $\begin{array}{l}\text { ovada, lanceo- } \\
\text { lada o elíptica }\end{array}$ \\
\hline Borde de la hoja & entero o dentado & dentado & entero \\
\hline Largo de los peciolos & $0.7-2.9 \mathrm{~mm}$ & $6-15 \mathrm{~mm}$ & 4-7 mm \\
\hline Epidermis de la hoja & lisa & ampulosa & ampulosa \\
\hline $\begin{array}{l}\text { Tamaño de los pelos del } \\
\text { envés }(\mathrm{mm})\end{array}$ & 1.5 & 1.0 & 0.5 \\
\hline Forma de la cúpula & $\begin{array}{l}\text { pateliforme a } \\
\text { poculiforme }\end{array}$ & hemisférica & hemisférica \\
\hline $\begin{array}{l}\text { Apice de las escamas de } \\
\text { las cúpulas maduras }\end{array}$ & agudo & redondeado & redondeado \\
\hline Forma de la bellota & $\begin{array}{l}\text { globosa a com- } \\
\text { primida }\end{array}$ & ovoide & ovoide \\
\hline Altitud (m s.n.m.) & $1300-1950$ & $1900-3000$ & $1900-2900$ \\
\hline Distribución & Mich. Méx. & $\begin{array}{l}\text { S.L.P. Qro. Hgo. } \\
\text { Mich. Méx. Tlax. } \\
\text { Mor. Pue. Ver. } \\
\text { Oax. Gro. }\end{array}$ & $\begin{array}{l}\text { S.L.P. Jal. Qro. } \\
\text { Hgo. Mich. Méx. } \\
\text { Tlax. Mor. Pue. } \\
\text { Ver. Chis. }\end{array}$ \\
\hline
\end{tabular}

Durante las visitas realizadas a los sitios que cita Warburg vimos que éstos son similares entre sí en cuanto a altitud así como a la cubierta vegetal, en su fisonomía, especies dominantes y asociadas.

Llamó la atención la amplia variación morfológica en los individuos del encino en cuestión de distintas edades, por ejemplo en los muy jóvenes las hojas son completamente glabras y suborbiculares y los troncos rugosos, en tanto que en los adultos las hojas presentan tomento abundante, su forma tiene gran variación y el tronco posee placas cuadrangulares.

También observamos que las hojas de renuevos y de algunas ramas que crecen a distintas alturas de tallos maduros de la especie estudiada son parecidas a las que se presentan en individuos jóvenes con tallas de $30 \mathrm{~cm}$ a $2 \mathrm{~m}$ de alto. Esta variabilidad en los caracteres morfológicos es la que se aprecia en las descripciones originales de las cuatro entidades y es la que Warburg utilizó para diferenciarlas. 
Acta Botánica Mexicana (1995), 31:63-71

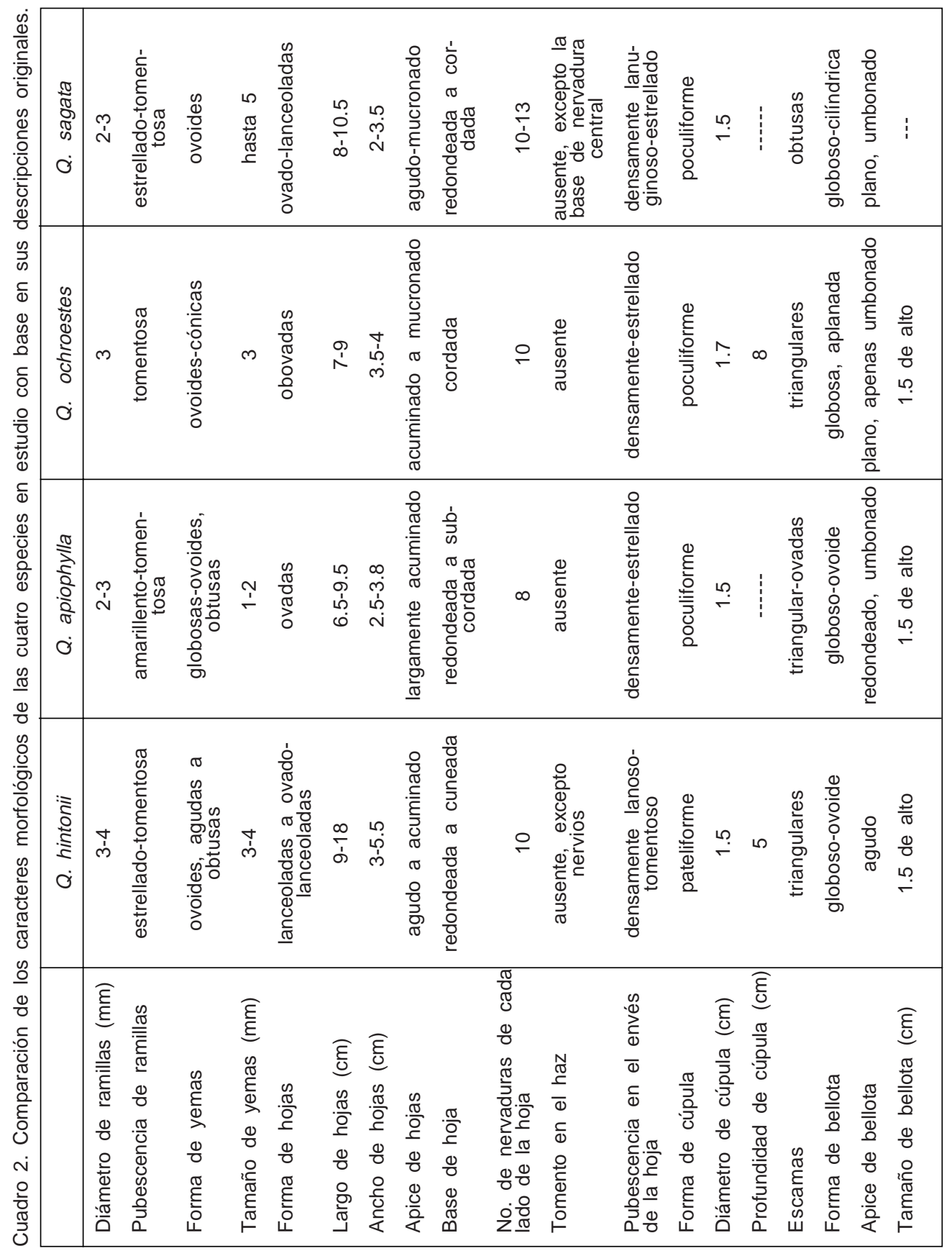


La falta de rasgos convincentes que separen a Quercus apiophylla, Q. ochroestes, $Q$. sagata y $Q$. hintonii nos hace pensar que todos corresponden a variantes de una sola especie, para la cual se propone este último binomio, conforme al artículo 57 del Código Internacional de Nomeclatura Botánica, Greuter et al. (1988), en donde se determina que el autor que primero reúne táxones portadores de nombres publicados con la misma fecha tiene derecho a elegir entre ellos. También se propone incluir a $Q$. hintonii en la serie Crassifoliae (Trelease, 1924) debido a su afinidad con Q. crassifolia y Q. dysophylla.

Quercus hintonii Warb., Kew Bull. 1939: 91. 1939. Tipo: MEXICO. México: Distrito de Temascaltepec, Nanchititla, julio de 1934, Hinton 6359 (K). Q. apiophylla Warb., Kew Bull. 1939: 95. 1939. Tipo: MEXICO. México: Distrito de Temascaltepec, Berros, septiembre de 1934, Hinton 6568 (K). Q. ochroestes Warb., Kew Bull. 1939: 94. 1939. Tipo: MEXICO. México: Mina de Agua, septiembre de 1934, Hinton 6576 (K). Q. sagata Warb., Kew. Bull. 1939: 90. 1939. Tipo: MEXICO. México: Distrito de Temascaltepec, Cuentla, septiembre de 1934, Hinton 6577 (K).

Arbol caducifolio, hasta de $15 \mathrm{~m}$ de alto; tronco de 30-50 cm de diámetro, corteza con placas cuadrangulares, en individuos muy jóvenes es rugosa; ramillas jóvenes de 1$4 \mathrm{~mm}$ de diámetro, con abundante tomento blanco-amarillento de pelos estrellados que se reduce y ennegrece con el tiempo, lenticelas blancas, de $0.5-1 \mathrm{~mm}$, más notorias en ramas viejas; yemas ovoides, de color castaño oscuro, de 1-8 mm de largo, con escamas coriáceas y pilosas; estípulas oblanceoladas, de $9-13 \mathrm{~mm}$ de largo, membranosas, pilosas en márgenes y base, deciduas; hojas jóvenes con abundante tomento rojizo que cambia a amarillo en la madurez, coriáceas, lanceoladas, ovado-lanceoladas, obovadas o elípticas, de 5-21 cm de largo y de 3-10 cm de ancho; ápice aristado, corta a largamente acuminado; base obtusa, redondeada, cordada o aguda, con frecuencia asimétrica; margen entero o aristado, muchas veces dentado, revoluto, con 7 o menos aristas en cada lado, es frecuente que éstas se presenten en un solo lado; las nervaduras se prolongan hasta las aristas, 6-14 en cada lado, rectas o ligeramente arqueadas, ascendentes, ramificándose en el borde; haz verde claro, lustroso, glabro excepto en la base y en la nervadura central, en hojas inmaduras es rojizo a verde oscuro, con abundante tomento formado por pelos estrellados y simples glandulares, con la madurez la pubescencia disminuye en cantidad y cambia de rojiza a blanco-amarillenta; nervadura central a veces algo elevada, nervaduras primarias y secundarias impresas; envés lanoso-tomentoso, con pelos estrellados de aproximadamente $1.5 \mathrm{~mm}$ de largo, con las ramas entrelazadas, epidermis lisa, con abundantes pelos simples glandulares, de color ámbar, el tomento cambia de blanco, en hojas jóvenes, a amarillo, en las maduras, nervaduras conspicuamente elevadas; pecíolo de $0.7-2.9 \mathrm{~cm}$ de largo y de 1-2 mm de ancho, lanoso-tomentoso, con frecuencia más ancho en la base; amentos masculinos de $3-10 \mathrm{~cm}$ de largo con muchas flores, raquis con abundante tomento, perianto pubescente de $1-2 \mathrm{~mm}$ de largo y de 2-3 $\mathrm{mm}$ de diámetro, amarillento, con frecuencia los bordes son rojos, estambres 6-7, exsertos, anteras oblongas, glabras, apendiculadas, de $1-1.5 \mathrm{~mm}$ de largo y $1 \mathrm{~mm}$ de ancho, con frecuencia rojizas, filamentos hasta de $2 \mathrm{~mm}$ de largo; amentos femeninos de 1-14 cm de largo, con 1-6 flores, raquis pubescente; perianto de $2 \mathrm{~mm}$ de largo y $1.5 \mathrm{~mm}$ de ancho, amarillento; estigmas 3-4, espatulados, de color rojo oscuro; frutos 1-4 en pedúnculos de 6-7.5 mm; cúpula poculiforme a pateliforme, de 10-14 $\mathrm{mm}$ de diámetro, de 4-7 $\mathrm{mm}$ de alto, escamas laxas con ápices 


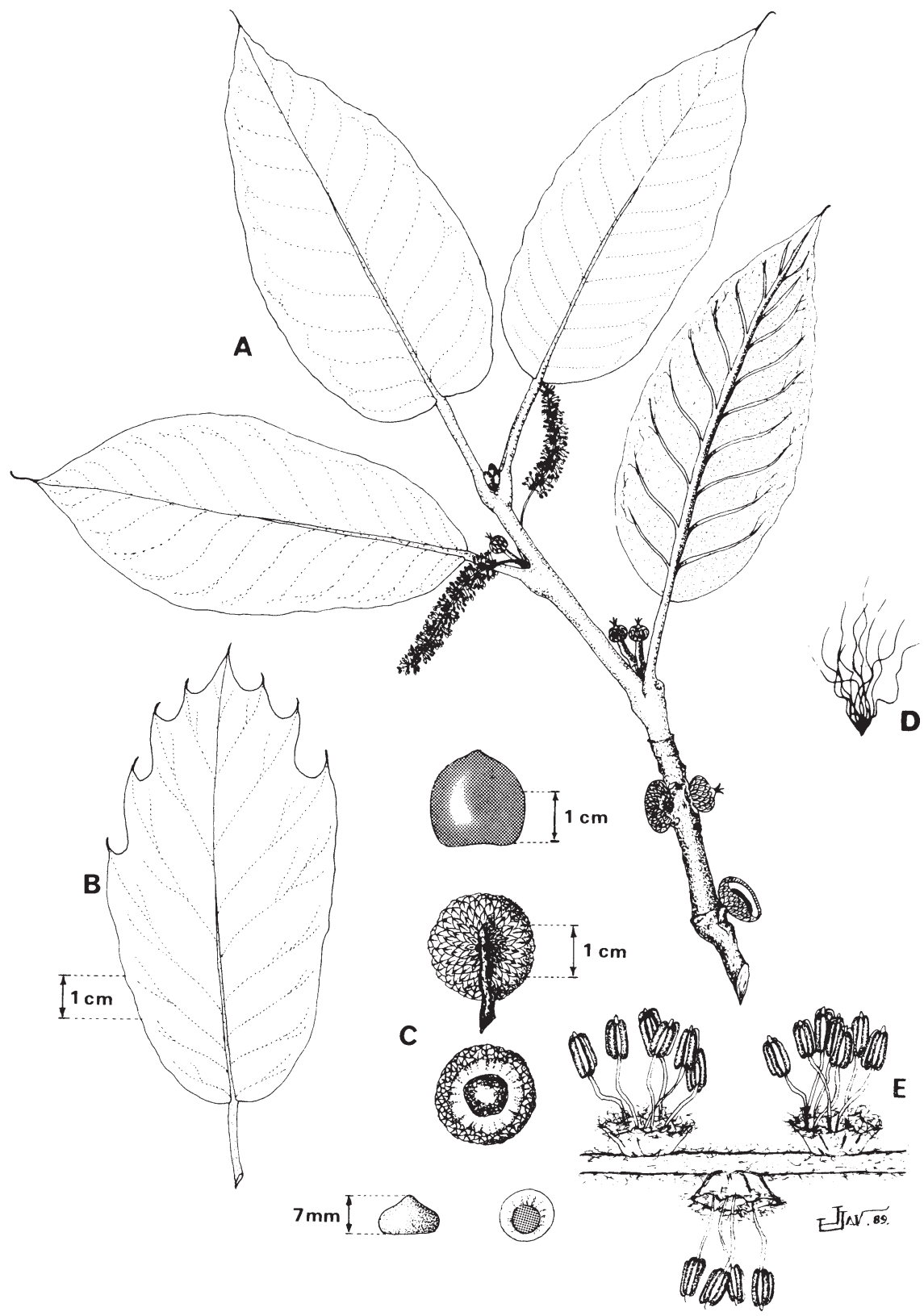

Fig. 1. Quercus hintonii. A. Rama con hojas, flores y frutos; B. Variación en hoja; C. Morfología de fruto; D. Pelos del envés de la hoja; E. Flores masculinas. 
agudos de color castaño, pubescentes; bellota globosa a comprimida, de 6-15 mm de largo, de 9-13 $\mathrm{mm}$ de diámetro con el ápice plano a umbonado (Fig. 1). Florece en marzo y fructifica de junio a octubre.

Material adicional examinado: MEXICO. México. Mpio. Amatepec, $4 \mathrm{~km}$ al $\mathrm{S}$ de Amatepec, Pineda R. 1052 (INIF); Mpio. Amatepec, Clachichilpan $7 \mathrm{~km}$ al NE, Pineda R. 1048 (INIF); Mpio. Amatepec y cercanías, Matuda \& Col. 29823 (CODAGEM); Mpio. San Simón de Guerrero, Mina de Agua, Rojas \& Romero 3605 (ENCB, IZTA); Mpio. San Simón de Guerrero, Los Berros Cuentla, Rojas \& Romero 3606 (IZTA, ENCB); Mpio. San Simón de Guerrero, km 83 Toluca-San Diego Cuentla, Rojas, Romero \& Aguilar 3218 (ENCB, IZTA); Mpio. Sultepec, km 25 carretera a San Miguel Totolmoloya, Torres Z. 575 (IZTA); Mpio. Tejupilco, alrededores de la población de Tejupilco, Rzedowski 30302 (INIF); Mpio. de Tejupilco, 5 km al NE de Palos Prietos, Pineda R. 1044 (INIF); Mpio. Tejupilco, 5 km al SW de Nanchititla, González M. 5026, 5049 (MEXU); Mpio. Tejupilco, Cerro de Nanchititla, Arcia s.n. (IZTA); Mpio. Tejupilco, Peña Bonita, González M. 5399, 5444, 5447 (MEXU); Mpio. Tejupilco, Potrero Chico, González M. 5436, 5448 (MEXU); Mpio. Tejupilco, Reserva Ecológica de Nanchititla, Jiménez F. 318 (IZTA); Mpio. Tejupilco, Los Cuervos, Rojas \& Romero 3604 (ENCB, IZTA); Mpio. Temascaltepec, km 70 Carr. Federal 134, Rojas \& Romero 3400 (ENCB, IZTA); Mpio. Temascaltepec, Chorrera, Hinton 6199 (ENCB); Mpio. Temascaltepec, Pantoja, Hinton 6225 (ENCB); Mpio. Temascaltepec, $5 \mathrm{~km}$ al SW de Temascaltepec, sobre la carretera a Tejupilco, Rzedowski 20829 (INIF); Mpio. Tlatlaya, Tlatlaya y cercanías, Matuda \& Col. 29825, 29827, 31588, (CODAGEM). Michoacán. Mpio. Villa Madero, sin localidad, ladera de Cerro, X. Madrigal 3140 (INIF).

Distribución y habitat: Estado de México: Municipios de Tejupilco, Temascaltepec, San Simón de Guerrero, Amatepec, Sultepec y Tlatlaya. Estado de Michoacán: Municipio de Villa Madero (Fig. 2). Se le encuentra en bosques de encino-pino con suelos rojizos, especies dominantes de estas comunidades son Quercus magnoliifolia, Q. hintonii y Pinus oocarpa; otros árboles asociados son Pinus pringlei, Clethra mexicana y Juniperus flaccida. En altitudes de 1300-1950 m s.n.m.

Nombre popular y usos: A esta especie se le llama encino prieto y se emplea localmente para la elaboración de mangos de herramientas, vigas, postes de cercas, y bancos rústicos, así como para leña. Podría utilizarse para fabricar duelas de parquet lambrín, muebles, durmientes, pilotes para minas y barricas (Arcia, 1979).

\section{AGRADECIMIENTOS}

Las autoras deseamos expresar nuestro agradecimiento al Biól. Luciano Vela Gálvez por su ayuda en la adquisición de los fototipos y las descripciones originales de las especies en estudio. Al Dr. J. Rzedowski por sus valiosas recomendaciones y sugerencias al presente trabajo. A la M. en C. Judith Espinosa por la revisión y observaciones al manuscrito. Al M. en C. Carlos Rojas Zenteno por su apoyo en la colecta del material botánico y sus sugerencias. Al M. en C. José Jaime Avila V. y al Biól. Martín Orozco V. por la elaboración de las ilustraciones. 
Acta Botánica Mexicana (1995), 31:63-71
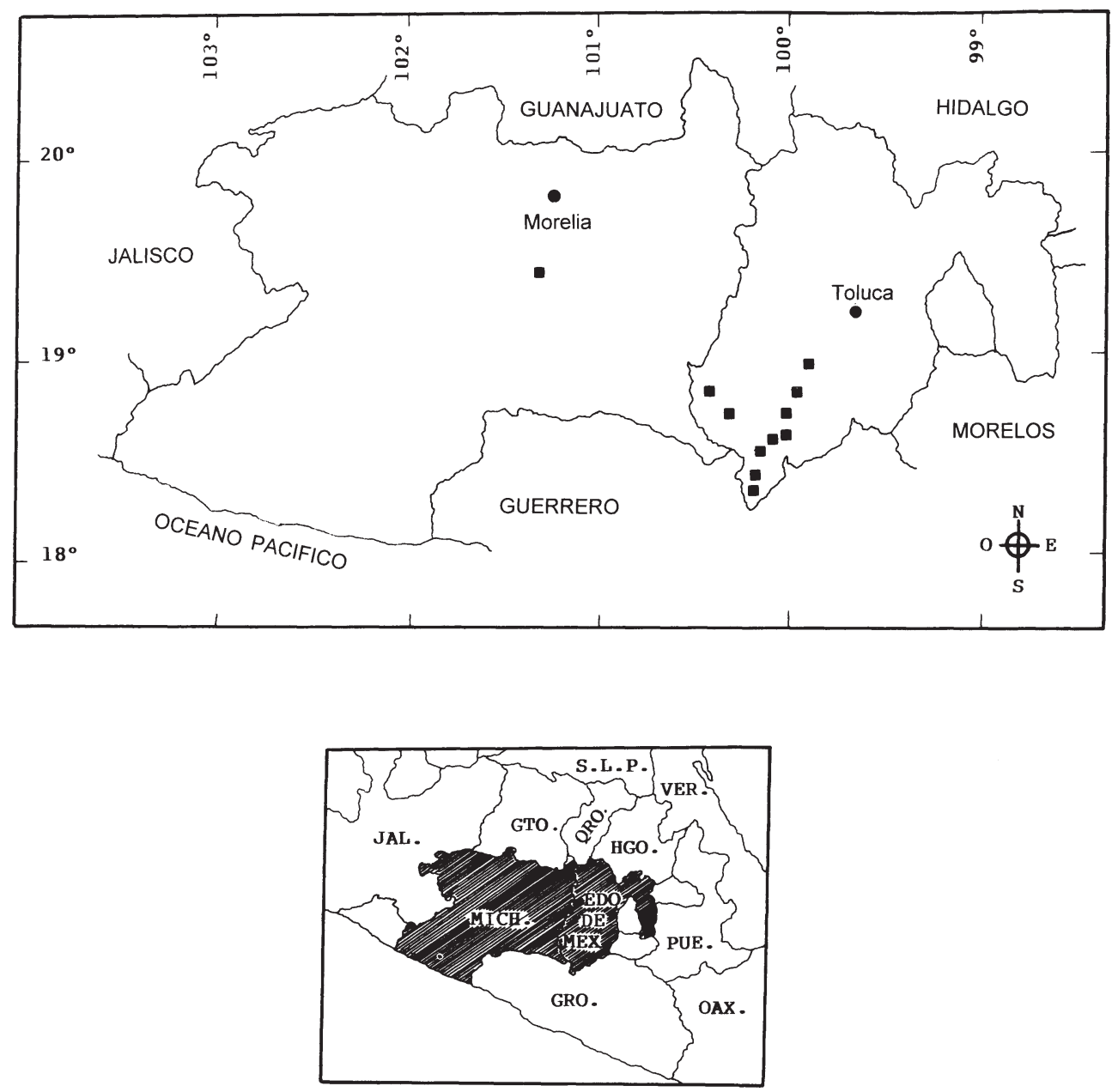

Fig. 2. Distribución geográfica de Quercus hintonii. 


\section{LITERATURA CITADA}

Arcia, G. 1979. Anatomía y características físicas de la madera de tres encinos del Estado de México. Tesis de Licenciatura. Escuela Nacional de Agricultura. Chapingo, México. 75 pp.

Greuter, W., H. M. Burdet, W. G. Chaloner, V. Demoulin, R. Grolle, D. L. Hawks-Worth, D. H. Nicholson, P. C. Silva, F. A. Stafleu, E. G. Voss \& J. McNeill. 1988. International code of botanical nomeclature. Koeltz Scientific Books. Konigstein. pp. 58-60.

Martínez, M. 1954. Los encinos del Estado de México. Comisión Botánico Exploradora del Estado de México. Gobierno del Estado de México, Dirección de Agricultura y Ganadería. Toluca, México. $77 \mathrm{pp}$.

Muller, C. H. y R. McVaugh. 1972. The oaks (Quercus) described by Née (1801), with comments on related species. Contr. Univ. Mich. Herb. 9(7): 507-522.

Trelease, W. 1924. The American oaks. Mem. Nat. Acad. Sci. 20: 38.

Warburg, E. 1939. The genus Quercus. "Plantae Mexicanae Hintonianae". Kew Bull. Misc. Inf. 1939: 84-95. 